\title{
Local Decomposition and Observability Properties for Automatic Calibration in Mobile Robotics
}

\author{
Agostino Martinelli
}

\begin{abstract}
This paper considers the problem of sensor selfcalibration in mobile robotics by only using a single point feature (e.g. a source of light). In particular, the problem of determining the extrinsic parameters of a bearing sensor mounted on a mobile platform (e.g. a camera) and simultaneously estimating the parameters describing the systematic error in the odometry system is discussed. Special attention is devoted to investigate the dependence of the observability properties of these parameters on the chosen robot trajectory.

The main contribution provided by this paper is the introduction of a new method to deal with estimation problems in the framework of mobile robotics. Specifically, a calibration problem has been considered. However, the same method can be adopted to solve other fundamental estimation problems. The method is based on the theory of distributions which exploits all the system Lie symmetries. Regarding the considered calibration problem this method allows analytically detecting the combinations of the calibration parameters which are observable for a given robot trajectory. Experiments are provided to validate the results.
\end{abstract}

\section{INTRODUCTION}

Calibration is the problem of estimating the parameters characterizing the systematic error of a sensor. In mobile robotics, performing this process on-line is not only a desire which automatizes a work which would have to be performed by hand, but it is in many cases a real need. This is especially true for the odometry. Indeed, the pressure of tires can change over time and the effective wheelbase depends on the terrain where the robot is moving. Having a system able to adapt continuously to different floor types and changing wheels attributes (i.e. different tire pressure, deterioration, etc.) is a key advantage.

Several strategies have been developed to perform online self-calibration. In many cases, an Extended Kalman Filter has been introduced to simultaneously estimate the robot configuration and the parameters characterizing the systematic error of a sensor (i.e., to solve the Simultaneous Localization and Auto Calibration $(S L A C)$ problem). Regarding the odometry, the $S L A C$ problem has been investigated in [4], [5], [8], [9], [14] and [16] both for indoor and outdoor environments. The same idea was adopted to self calibrate a vision sensor [11]. In this work, an observability analysis taking into account the system nonlinearities was also provided to understand whether the system contains the necessary information to perform the self calibration. Indeed,

A. Martinelli is with INRIA Rhone Alpes, Grenoble, France agostino.martinelli@inrialpes.fr

This work was supported by the European Commission FP7-ICT-20073.2.2 Cognitive Systems, Interaction, and Robotics under the contract \#231855 (sFLY) and the EU Integrated Projects BACS - FP6-IST-027140 a necessary condition to perform the estimation of a state, is that the state is observable. In [11] it was investigated whether the state containing the extrinsic parameters of the vision sensor is or is not observable. The observability rank criterion introduced by Hermann and Krener [6] was adopted to this scope. The observability rank criterion has also been adopted in cooperative localization [10] and in SLAM [13]. Regarding calibration problems, it has also been applied for the odometry self-calibration [12]. However, in all these works what it was determined is only whether the state defining the system configuration is observable or not.

In this paper we want to do a step forward whose importance is in our opinion fundamental when dealing with a non observable problem. Indeed, when a state is not observable, estimating directly the state brings to inconsistency with catastrophic consequences. On the other hand, when a state is not observable, suitable combinations of its components could be observable and therefore could be estimated. The derivation of these combinations is very important in order to properly exploit the information contained in the sensor data to estimate a given set of parameters. This derivation requires to perform a local decomposition [7] which exploits all the system Lie symmetries. While in the linear case this decomposition is easy to be done, in the non linear case it is often troublesome. In this paper we apply such approach to the problem of self-calibration in mobile robotics. We consider the case when the calibration is carried out by only using a single point feature. In particular, we consider the following calibration problems:

- The extrinsic self calibration of a bearing sensor (i.e. the same problem considered in [11]);

- The simultaneous self calibration of the odometry system and the extrinsic calibration of a bearing sensor (to the best of our knowledge this problem has never been investigated before).

In both cases we perform a local decomposition as illustrated in [7] in order to analytically derive which combinations of the parameters are observable. In particular, we derive these combinations for several different contexts, basically defined by the robot trajectory.

In section II we define the considered systems. In section III we remind some results from [6] and [7]. In section IV and $\mathrm{V}$ we perform the decomposition for different robot trajectories respectively in the two cases mentioned before. This allows us to detect for every robot trajectory the combinations of the parameters which are observable. A validation is given in section VI where experimental results 
are shown. Finally, conclusions are provided in section VII.

\section{THE CONSIDERED SYSTEMS}

We consider a mobile robot moving in a $2 D$-environment. The configuration of the robot in a global reference frame can be characterized through the vector $X=\left[x_{R}, y_{R}, \theta_{R}\right]^{T}$ where $x_{R}$ and $y_{R}$ are the cartesian robot coordinates and $\theta_{R}$ is the robot orientation. The dynamics of this vector are described by the following non-linear differential equations:

$$
\dot{X}=f(X, u)=\left[\begin{array}{l}
\dot{x}_{R}=v \cos \theta_{R} \\
\dot{y}_{R}=v \sin \theta_{R} \\
\dot{\theta}_{R}=\omega
\end{array}\right.
$$

where $v$ and $\omega$ are the linear and the rotational robot speed, respectively. The link between these velocities and the robot controls $u$ depends on the considered robot drive system. We will consider the case of a differential drive. In order to characterize the systematic odometry error we adopt the model introduced in [3]. We have:

$$
v=\frac{\delta_{R} v_{R}+\delta_{L} v_{L}}{2} \quad \omega=\frac{\delta_{R} v_{R}-\delta_{L} v_{L}}{\delta_{B} B}
$$

where $v_{R}$ and $v_{L}$ are the control velocities (i.e. $u=$ $\left[v_{R}, v_{L}\right]^{T}$ ) for the right and the left wheel, $B$ is the nominal value for the distance between the robot wheels and $\delta_{R}, \delta_{L}$ and $\delta_{B}$ characterize the systematic odometry error due to an uncertainty on the wheels diameters and on the distance between the wheels.

Furthermore, a bearing sensor is mounted on the robot. We assume that the camera's optical axis is aligned with the $z$-axis of the robot reference frame and therefore the transformation between the camera and robot reference frames is characterized through the three parameters $\phi, \rho$ and $\psi$ (see fig. 1). In the case we consider an omnidirectional mirror camera, we assume that this axis is aligned with the mirror's axis.

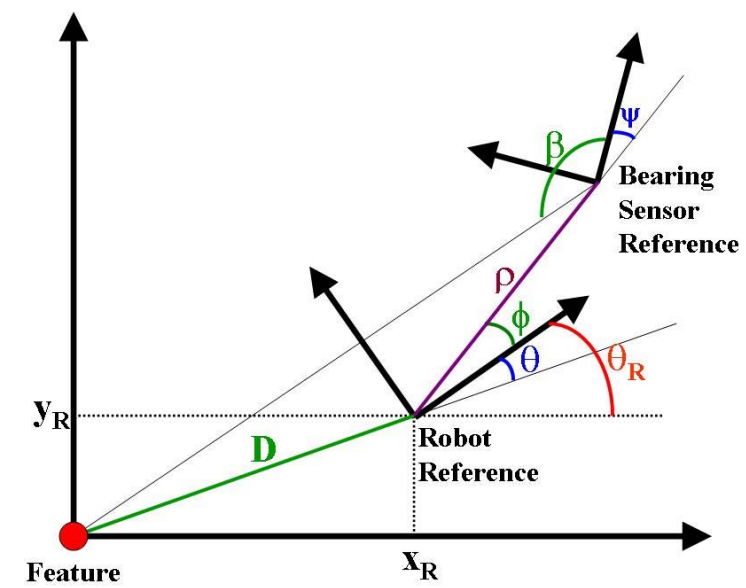

Fig. 1. The two reference frames respectively attached to the robot and the bearing sensor.
The available data are the control $u=\left[v_{R}, v_{L}\right]^{T}$ and the bearing angle of a source of light ( $\beta$ in fig. 1) at several time steps during the robot motion.

We introduce the following quantities:

$$
\begin{gathered}
\theta \equiv \theta_{R}-\operatorname{atan} 2\left(y_{R}, x_{R}\right) ; \quad \gamma \equiv \theta+\phi ; \\
\mu \equiv \frac{\rho}{D} \equiv \frac{\rho}{\sqrt{x_{R}^{2}+y_{R}^{2}}}
\end{gathered}
$$

By using simple trigonometry algebra we obtain (see also fig. 1):

$$
\beta=-\operatorname{atan}\left(\frac{\sin \gamma}{\mu+\cos \gamma}\right)-\psi+\left(1+S_{p}\right) \frac{\pi}{2}
$$

where $S_{p}$ can be \pm 1 depending on $\gamma$. In particular, $S_{p}=1$ when $\gamma_{-} \leq \gamma \leq \gamma_{+}$and $S_{p}=-1$ when $\gamma>\gamma_{+}$or $\gamma<$ $\gamma_{-}, \gamma_{-}$and $\gamma_{+}$being the two solutions (in $[-\pi, \pi)$ ) of the equation $\cos \gamma=-\mu$ with $\gamma_{+}=-\gamma_{-}$and $\gamma_{+}>0$. We made the assumption $0<\mu<1$ since we want to avoid collisions between the robot and the feature $(D>\rho)$.

\section{A. Camera Self-Calibration}

In this case we assume that the odometry is calibrated. As a result, knowing $u=\left[v_{R}, v_{L}\right]^{T}$ means knowing $[v, \omega]^{T}$. The goal is to estimate on-line the three parameters $\phi, \rho$ and $\psi$ by using the available data (i.e. $v, \omega$ and $\beta$ in a given time interval). Our system is described by the following equations:

$$
\left\{\begin{array}{l}
\dot{\mu}=-\mu^{2} \frac{v}{\rho} \cos (\gamma-\phi) \\
\dot{\gamma}=\omega-\mu \frac{v}{\rho} \sin (\gamma-\phi) \\
\dot{\phi}=\dot{\rho}=\dot{\psi}=0
\end{array}\right.
$$

In section IV we derive for several robot trajectories, which combinations of $\mu, \gamma, \phi, \rho$ and $\psi$ are observable and hence can be estimated.

\section{B. Simultaneous Camera and Odometry Self-Calibration}

In this case $v$ and $\omega$ are not directly provided by $v_{R}$ and $v_{L}$. The goal is to estimate simultaneously the parameters $\phi$, $\rho, \psi, \delta_{R}, \delta_{L}$ and $\delta_{B}$ using the available data (i.e. $v_{R}, v_{L}$ and $\beta$ in a given time interval). Since these data consists of angle measurements (the wheel diameters are not known and in fact are to be estimated), the best we can hope is the possibility to estimate these parameters up to a scale factor. In particular, we will refer to the following parameters: $\phi$, $\psi, \eta \equiv \frac{\delta_{R}}{2 \rho}, \delta \equiv \frac{\delta_{L}}{\delta_{R}}$ and $\xi \equiv \frac{1}{B} \frac{\delta_{R}}{\delta_{B}}$.

The dynamics of our system are described by the following equations:

$$
\left\{\begin{array}{l}
\dot{\mu}=-\mu^{2} \eta\left(v_{R}+\delta v_{L}\right) \cos (\gamma-\phi) \\
\dot{\gamma}=\xi\left(v_{R}-\delta v_{L}\right)-\mu \eta\left(v_{R}+\delta v_{L}\right) \sin (\gamma-\phi) \\
\dot{\phi}=\dot{\psi}=\dot{\eta}=\dot{\delta}=\dot{\xi}=0
\end{array}\right.
$$

In section $\mathrm{V}$ we derive for several robot trajectories, which combinations of $\mu, \gamma, \phi, \psi, \eta, \delta$ and $\xi$ are observable and hence can be estimated. 
Both the dynamics in (5) and in (6) have the following structure:

$$
\dot{X}=f(X, u)=\sum_{i=0}^{M} f_{i}(X) u_{i}
$$

with $M=2$ in both cases. Furthermore, in (5) we have: $X=[\mu, \gamma, \phi, \rho, \psi]^{T}, u_{1}=v, u_{2}=\omega$ and

$$
\begin{aligned}
& f_{1}=\left[-\frac{\mu^{2}}{\rho} \cos (\gamma-\phi),-\frac{\mu}{\rho} \sin (\gamma-\phi), 0,0,0\right]^{T} \\
& f_{2}=\left[\begin{array}{llll}
0, & 1,0,0,0
\end{array}\right]^{T}
\end{aligned}
$$

while in (6) we have: $X=[\mu, \gamma, \phi, \psi, \eta, \delta, \xi]^{T}$, $u_{1}=v_{R}, u_{2}=v_{L}$ and

$$
\begin{aligned}
& f_{1}=-\left[\mu^{2} \eta \cos (\gamma-\phi), \mu \eta \sin (\gamma-\phi)-\xi, 0,0,0,0,0\right]^{T} \\
& f_{2}=-\delta\left[\mu^{2} \eta \cos (\gamma-\phi), \mu \eta \sin (\gamma-\phi)+\xi, 0,0,0,0,0\right]^{T}
\end{aligned}
$$

\section{OBSERVABILITy PRoperties AND LOCAL DECOMPOSITION}

In control theory, a system is defined as observable when it is possible to reconstruct its initial state by knowing, in a given time interval, the control inputs and the outputs [7]. The observability property has a very practical meaning. When a system is observable it contains all the necessary information to perform the estimation with an error which is bounded [7].

\section{A. Observability Rank Criterion}

In a nonlinear system the concept of local distinguishability was introduced by Hermann and Krener [6]. The same authors introduced also a criterion, the observability rank criterion, to verify whether a system has this property. This criterion plays a very important role since in many cases a nonlinear system, whose associated linearized system is not observable, has however the local distinguishability property. Regarding the localization problem this was proven in [1] and [2]. Note that it is the distinguishability property telling us that the system contains the necessary information to have a bounded estimation error (actually, provided that the locality is large enough with respect to the sensor accuracy).

We now want to remind some concepts in the theory by Hermann and Krener in [6]. We will adopt the following notation. We indicate the $K^{\text {th }}$ order Lie derivative of a field $\Lambda$ along the vector fields $v_{i_{1}}, v_{i_{2}}, \ldots, v_{i_{K}}$ with $L_{v_{1}, v_{i_{2}}, \ldots, v_{i_{K}}}^{K} \Lambda$. Note that the Lie derivative is not commutative. In particular, in $L_{v_{i_{1}}, v_{i_{2}}, \ldots, v_{i_{K}}}^{K} \Lambda$ it is assumed to differentiate along $v_{i_{1}}$ first and along $v_{i_{K}}$ at the end.

Let us indicate with $\Omega$ the space spanned by all the Lie derivatives $\left.L_{f_{i_{1}}, f_{i_{2}}, \ldots, f_{i_{K}}}^{K} \beta\right|_{t=0}\left(i_{1}, i_{2}, \ldots, i_{K}=1,2\right.$ and the functions $f_{i_{j}}$ are defined in (9).

Furthermore, we denote with $d \Omega$ the space spanned by the gradients of the elements of $\Omega$.
In this notation, the observability rank criterion can be expressed in the following way: The dimension of the $o b$ servable sub-system at a given $X_{0}$ is equal to the dimension of $d \Omega$.

In sections IV and V we adopt this criterion to investigate the observability properties of the two systems introduced respectively in section II-A and II-B. In particular, we evaluate the dimension of $d \Omega$ for several trajectories. When the dimension of $d \Omega$ is smaller than the dimension of the entire configuration space, we perform a local decomposition of the system.

\section{B. Local Decomposition}

Let us suppose that a given system is not observable. We refer to the case where the dynamics have the structure as in (7) but with a single input control. Indeed, in the next section we consider trajectories with one degree of freedom. By denoting the state with $S$, we can describe the system by the following equations:

$$
\left\{\begin{array}{l}
\dot{S}=f(S) u, \quad S \in \mathbb{R}^{n} \\
y=h(S)
\end{array}\right.
$$

where $y$ is the output (observation) of the system (in our case $y=\beta \in \mathbb{R}$ )

We are assuming that $S$ is not observable, i.e. the dimension of the associated subspace $d \Omega$ is smaller than $n$ (in this case the Lie derivatives are computed only along the vector field $f(S)$ ). Let us suppose that this dimension is equal to $n_{o b s}$. According to the theory of distributions developed in [7], we can find $n_{o b s}$ independent combinations of the components of the original state $S$ which are observable and $n-n_{o b s}$ independent combinations of the components of $S$ which are not observable. More precisely, if we include the $n_{o b s}$ observable combinations in the vector $S_{2}$ and the other $n-n_{o b s}$ combinations in the vector $S_{1}$, we have the following decomposition for the original system:

$$
\left\{\begin{array}{l}
\dot{S}_{1}=f_{1}\left(S_{1}, S_{2}\right) u \\
\dot{S}_{2}=f_{2}\left(S_{2}\right) u \\
y=h_{2}\left(S_{2}\right)
\end{array}\right.
$$

In particular, the subsystem defined by the last two equations in (11) is independent of the value of $S_{1}$ and it is observable. Therefore, by performing this decomposition, we can use the information coming from the dynamics (i.e. the knowledge of $u(t))$ and the observations $(y(t))$ in order to estimate the observable quantities $\left(S_{2}\right)$. This decomposition is very important in every estimation problem when the state is not observable. Indeed, estimating directly the original state $S$ results in an erroneous evaluation.

In section II-A, when we introduced the two quantities defined in (3), we performed such a decomposition for the state $\left[x_{R}, y_{R}, \theta_{R}, \phi, \rho, \psi\right]^{T}$ : indeed, the new state $[\mu, \theta, \phi, \rho, \psi]^{T}$ is observable as proven in [11] and its components are non linear combinations of the components of the original state (which is not observable). On the other hand, in the most of cases it is very troublesome to perform such a decomposition. 
In the next two sections we perform such a decomposition in several contexts. In two cases we apply the distributions theory developed in [7].

\section{ObSERVABILITy PRoperties AND Local} DECOMPOSITION FOR THE SYSTEM DEFINED IN SECTION II-A

We consider the following four cases:

1) Pure rotations and far feature;

2) Pure shift and far feature;

3) Pure rotations and close feature;

4) Pure shift and close feature.

In the following, with far feature we mean that the value of $\rho$ is negligible $(\rho \rightarrow 0)$ and not necessarily that the distance of the feature $(D)$ is infinite. In particular, the distance traveled by the robot is not negligible with respect to $D$. In this limit we have:

$$
\lim _{\rho \rightarrow 0} \beta=\beta_{c} \equiv-\gamma-\psi
$$

Furthermore, for the system defined in section II-A, the pure rotation motion is defined by setting $v=0$ and $\omega \neq 0$, while the pure shift motion is defined by $v \neq 0$ and $\omega=0$.

\section{A. Pure Rotation and Far Feature}

The Lie derivatives of the observation function in (12) must be calculated only along the vector $f_{2}$ in (8). It is easy to realize that the dimension of $d \Omega$ is 1 (the first order Lie derivative is equal to -1 , i.e. is a constant). This result is intuitive: the observation in (12) does not provide any information on $\mu$ and $\rho$ and it is not able to distinguish among $\gamma$ and $\psi$. Furthermore, the pure rotation does not provide any additional information. Hence, it is only possible to observe the sum $\gamma+\psi$. The observable part of the local decomposition in this case is given by the equations:

$$
\left\{\begin{array}{l}
\dot{\alpha}=\omega \\
y=-\alpha
\end{array}\right.
$$

where $\alpha \equiv \gamma+\psi$ is the only observable quantity.

\section{B. Pure Shift and Far Feature}

The Lie derivatives of the function in (12) must be calculated only along the vector $f_{1}$ in (8). By adopting the variables $D=\frac{\rho}{\mu}, \theta=\gamma-\phi$ and $\zeta \equiv \phi+\psi$ instead of the original state $[\mu, \gamma, \phi, \rho, \psi]^{T}$, we obtain the following equations:

$$
\left\{\begin{aligned}
\dot{D} & =v \cos \theta \\
\dot{\theta} & =-\frac{v}{D} \sin \theta \\
\dot{\zeta} & =0 \\
y & =-\theta-\zeta
\end{aligned}\right.
$$

By applying the observability rank criterion (see the appendix), it is easy to prove that the state $[D, \theta, \zeta]$ is observable. Therefore, the system described by (14) represents the observable part of a local decomposition for the original system (when the feature is far and $\omega=0$ ). In other words, the state $[D, \theta, \zeta]$ plays the role of $S_{2}$ in (11).

\section{Pure Rotation and Close Feature}

In this case the expression for the observation is the one given in (4). Furthermore, $v=0$. The expression in (4) depends only on $\mu, \gamma$ and $\psi$. Furthermore, $f_{2}$ in (8) is independent of the state. Therefore, the best we can hope is that these three quantities are observable. By directly computing the Lie derivatives and by applying the observability rank criterion it is possible to show that this is the case. Therefore, the observable part of the local decomposition is given by the equations:

$$
\left\{\begin{array}{l}
\dot{\mu}=0 \\
\dot{\gamma}=\omega \\
\dot{\psi}=0 \\
y=-\operatorname{atan}\left(\frac{\sin \gamma}{\mu+\cos \gamma}\right)-\psi+\left(1+S_{p}\right) \frac{\pi}{2}
\end{array}\right.
$$

\section{Pure Shift and Close Feature}

This is a very troublesome case. A priori, it is not possible to exclude that the entire state $[\mu, \gamma, \phi, \rho, \psi]^{T}$ is observable. However, a direct computation of the dimension of $d \Omega$ shows that this dimension is smaller than 5 . Furthermore, by applying the method introduced in [7], it is possible to detect the following observable quantities: $\chi_{1} \equiv \frac{\sin (\gamma+\psi)+\mu \sin \psi}{\mu \cos \psi+\cos (\gamma+\psi)}$, $\chi_{2} \equiv \frac{\mu}{\rho(\sin (\gamma+\psi)+\mu \sin \psi)}$ and $\chi_{3} \equiv \phi+\psi$. In particular, the observable part of the local decomposition is given by the equations:

$$
\left\{\begin{aligned}
\dot{\chi}_{1} & =v\left(\sin \chi_{3}-\chi_{1} \cos \chi_{3}\right) \chi_{1} \chi_{2} \\
\dot{\chi}_{2} & =-v \chi_{2}^{2} \sin \chi_{3} \\
\dot{\chi}_{3} & =0 \\
y & =-\operatorname{atan} \chi_{1}+k_{\pi}
\end{aligned}\right.
$$

where $k_{\pi}$ can be 0 or $\pi$ depending on the system parameters. We do not provide here neither the derivation of the previous result (i.e. the application of the method described in [7] for the considered system) nor the dependence of $k_{\pi}$ on the parameters. Indeed, the computation is troublesome in contrast with the result which has a very simple analytical expression. It is in any case easy to verify the validity of the equations in (16) by just performing differentiation. They represent a local decomposition since these equations only depend on the three quantities $\chi_{1}, \chi_{2}$, and $\chi_{3}$ and not on the original five variables $\mu, \gamma, \phi, \rho$ and $\psi$. Furthermore, by applying the rank criterion on the system in (16), it is possible to show that the dimension of the associated $d \Omega$ is equal to three.

\section{OBSERVABILITy PROPERTIES AND LOCAL DECOMPOSITION FOR THE SYSTEM DEFINED IN SECTION II-B}

We consider the same four cases of trajectory defined at the beginning of section IV. However, in this case instead of 
the pure rotation we define the motion obtained by setting $v_{R}=-v_{L} \equiv \nu$. Since the parameter $\delta$ is in general different from 1 , the motion actually accomplished by the robot with the previous control settings does not correspond to a pure rotation. However, since $\delta$ is unknown, we consider this motion instead of a true pure rotation. We will refer to this motion as Opposite Speeds Motion. Likewise, instead of the pure shift we define the motion obtained by setting $v_{R}=v_{L} \equiv \nu$. Again, this actually does not correspond to a shift in the $2 D$-space. We will refer to this motion as Equal Speeds Motion.

\section{A. Opposite Speeds Motion and Far Feature}

Let us start by considering the case of far feature. We will derive a local decomposition without the need of directly applying the method in [7]. The observation is provided in (12), which is independent of $\mu$. On the other hand, it is possible to reduce the number of variables in the equations in (6) by considering the new variable $\mu_{\eta} \equiv \mu \eta$. In other words, the system with the dynamics given in (6) and the observation given in (12) can be described by the following equations:

$$
\left\{\begin{aligned}
\dot{\mu}_{\eta} & =-\mu_{\eta}^{2}\left(v_{R}+\delta v_{L}\right) \cos \theta \\
\dot{\theta} & =\xi\left(v_{R}-\delta v_{L}\right)-\mu_{\eta}\left(v_{R}+\delta v_{L}\right) \sin \theta \\
\dot{\delta} & =\dot{\xi}=\dot{\zeta}=0 \\
y & =-\theta-\zeta
\end{aligned}\right.
$$

By directly computing the rank criterion it is possible to verify that the 5 variables of this system $\left(\mu_{\eta}, \theta, \delta, \xi\right.$ and $\zeta)$ are observable and therefore the system in (17) represents the observable part of a local decomposition for the original system when the feature is far.

On the other hand, when we consider the one-degree of freedom motion previously defined by the opposite speeds motion, we can further reduce the number of variables. By introducing the quantities $\mu_{-} \equiv \mu \eta(1-\delta)$ and $\xi_{+} \equiv \xi(1+\delta)$ we have:

$$
\left\{\begin{array}{l}
\dot{\mu}_{-}=-\mu_{-}^{2} \nu \cos \theta \\
\dot{\theta}=\xi_{+} \nu-\mu_{-} \nu \sin \theta \\
\dot{\xi}_{+}=\dot{\zeta}=0 \\
y=-\theta-\zeta
\end{array}\right.
$$

By directly computing the rank criterion it is possible to verify that the 4 variables of this system $\left(\mu_{-}, \theta, \xi_{+}\right.$and $\left.\zeta\right)$ are observable and therefore the system in (18) represents the observable part of a local decomposition for the original system when the feature is far and the robot accomplishes the opposite speeds motion.

\section{B. Equal Speeds Motion and Far Feature}

Similarly to the previous case, it is possible to verify that the observable quantities are in this case: $\mu_{+} \equiv \mu \eta(1+$ $\delta), \theta, \xi_{-} \equiv \xi(1-\delta)$ and $\zeta$. In particular, the equations for these observable subsystem are:

$$
\left\{\begin{array}{l}
\dot{\mu}_{+}=-\mu_{+}^{2} \nu \cos \theta \\
\dot{\theta}=\xi_{-} \nu-\mu_{+} \nu \sin \theta \\
\dot{\xi}_{-}=\dot{\zeta}=0 \\
y=-\theta-\zeta
\end{array}\right.
$$

\section{Opposite Speeds Motion and Close Feature}

We cannot eliminate $\mu$ as in the previous case since the observation depends on it. However, for the opposite speeds motion we can still have a reduction of variables for the system whose dynamics are given in (6) and the observation in (4). Indeed, we can easily eliminate $\delta$ by using $\xi_{+}$as in the previous case and $\eta_{-} \equiv \eta(1-\delta)$. We obtain:

$$
\left\{\begin{array}{l}
\dot{\mu}=-\mu^{2} \eta_{-} \nu \cos (\gamma-\phi) \\
\dot{\gamma}=\xi_{+} \nu-\mu \eta_{-} \nu \sin (\gamma-\phi) \\
\dot{\eta}_{-}=\dot{\xi}_{+}=\dot{\phi}=\dot{\psi}=0
\end{array}\right.
$$

The state defining this system is $\left[\mu, \gamma, \eta_{-}, \xi_{+}, \phi, \psi\right]^{T}$ and has dimension equal to 6 . By applying the rank criterion it is possible to find that the dimension of the associated $d \Omega$ is equal to 4 . Therefore, this is not a local decomposition for the original system. In particular, according to the theory developed in [7] it must be possible to detect 4 independent quantities which are non linear combinations of the original 6-dimensional state. We do not provide here the derivation (i.e. the application of the method described in [7] for the considered system). Indeed, the computation is very troublesome in contrast with the result which has a very simple analytical expression. We found the following observable quantities: $G \equiv \psi-\operatorname{atan} \Psi_{3} ; \quad L \equiv \psi-\operatorname{atan} \Psi_{1}^{-} ; \quad V \equiv$ $\Psi_{2}^{-} \frac{1+\Psi_{1}^{-} \Psi_{3}}{1+\Psi_{3}^{2}} ;$ and $\xi_{+}$

where:

$\Psi_{1}^{-} \equiv \frac{\xi_{+}-\eta_{-} \sin \phi}{\eta_{-} \cos \phi} ; \quad \Psi_{2}^{-} \equiv \frac{\mu \eta_{-} \cos \phi}{\sin \gamma} ; \quad \Psi_{3} \equiv \frac{\mu+\cos \gamma}{\sin \gamma}$

The local decomposition is:

$$
\left\{\begin{array}{l}
\dot{G}=\nu\left(-V+\xi_{+}\right) \\
\dot{V}=\nu V \tan (G-L)\left(-\xi_{+}+2 V\right) \\
\dot{L}=\dot{\xi}_{+}=0 \\
y=-G+S_{p} \frac{\pi}{2}
\end{array}\right.
$$

It is possible to verify the validity of the equations in (22) by just performing differentiation. They represent a local decomposition since these equations only depend on the 4 quantities $G, V, L$ and $\xi_{+}$and not on the original 6 dimensional state $\left[\mu, \gamma, \eta_{-}, \xi_{+}, \phi, \psi\right]^{T}$. Furthermore, by applying the rank criterion on the system in (22), it is possible to show that the dimension of the associated $d \Omega$ is equal to 4 . 


\begin{tabular}{|c|c|c|c|c|}
\hline Traj. & Far Rot & Far Shift & Close Rot & Close Shift \\
\hline Obs. & $\begin{array}{c}\alpha= \\
\gamma+\psi\end{array}$ & $\begin{array}{c}D=\frac{\rho}{\mu} \\
\theta=\gamma-\phi \\
\zeta=\phi+\psi\end{array}$ & $\begin{array}{l}\mu \\
\gamma \\
\psi\end{array}$ & $\begin{array}{c}\chi_{1}=\frac{\sin (\gamma+\psi)+\mu \sin \psi}{\mu \cos \psi+\cos (\gamma+\psi)} \\
\chi_{2}=\frac{\mu_{\mu}(\sin (\gamma+\psi)+\mu \sin \psi)}{\rho(\gamma+\psi} \phi \\
\chi_{3}=\phi+\psi\end{array}$ \\
\hline Eq. & Eq. (13) & Eq. (14) & Eq. (15) & Eq. (16) \\
\hline
\end{tabular}

\section{Equal Speeds Motion and Close Feature}

The result is exactly the same as in the previous case by defining $\Psi_{1}^{+}$and $\Psi_{2}^{+}$as $\Psi_{1}^{-}$and $\Psi_{2}^{-}$with the substitution of $\xi_{-}$instead of $\xi_{+}$and $\eta_{+} \equiv \eta(1+\delta)$ instead of $\eta_{-}$:

$$
\Psi_{1}^{+} \equiv \frac{\xi_{-}-\eta_{+} \sin \phi}{\eta_{+} \cos \phi} ; \quad \Psi_{2}^{+} \equiv \frac{\mu \eta_{+} \cos \phi}{\sin \gamma}
$$

\section{DISCUSSION AND EXPERIMENTS}

We summarize the results obtained in the previous two sections. Let us start with the results obtained in section IV. The system is defined in section II-A whose state is given by $[\mu, \gamma, \phi, \rho, \psi]^{T}$. In table I we provide a summary of the results obtained in section IV. In the first line we report all the considered trajectories. We provide all the independent quantities which are observable for all the considered trajectories in the second line. Finally, we indicate the equations to be adopted to implement a filter for their estimation in the third line.

Regarding the system defined in section II-B the results are summarized in table II. The state defining the system is in this case $[\mu, \gamma, \phi, \psi, \eta, \delta, \xi]^{T}$.

\section{A. Experiments}

For the experiments we adopted a mobile robot with a differential drive system equipped with encoder sensors on the wheels. In this preliminary experiment we consider only the problem of the extrinsic camera calibration (Indeed, the odometry system was previously calibrated). We equipped the robot with an omnidirectional vision sensor consisting of a webcam and a conic mirror (see fig $2 a$ ). Furthermore, we put on the origin a source of light consisting of a set of LEDs as shown in fig. $2 b$. Finally, we adopted the same strategy introduced in [15] to detect the source of light from the image based on the defocusing. The source of light appears as shown in fig. $2 c$.

According to the results provided in table I, it is possible to estimate the parameters $\phi, \rho$ and $\psi$ by combining a shift motion far from the feature with pure rotations close to the feature. Indeed, by performing the first trajectory, it is possible to observe the sum $\phi+\psi, D$ and $\theta$. Once the robot starts to rotate, $D$ does not change. Furthermore, the sum

\begin{tabular}{|c|c|c|}
\hline Traj. & Far Opposite Speeds & Far Equal Speeds \\
\hline Obs. & $\begin{array}{c}\mu_{-}=\mu \eta(1-\delta) \\
\theta=\gamma-\phi \\
\xi_{+}=\xi(1+\delta) \\
\zeta=\phi+\psi\end{array}$ & $\begin{array}{c}\mu_{+}=\mu \eta(1+\delta) \\
\theta=\gamma-\phi \\
\xi_{-}=\xi(1-\delta) \\
\zeta=\phi+\psi\end{array}$ \\
\hline Eq. & Eq. (18) & Eq. (19) \\
\hline $\begin{array}{l}\text { Traj. } \\
\text { Taj }\end{array}$ & Close Opposite Speeds & $\overline{\text { Close Equal Speeds }}$ \\
\hline Obs. & $\begin{array}{c}G=\psi-\operatorname{atan} \Psi_{3} \\
L=\psi-\operatorname{atan} \Psi_{1}^{-} \\
V=\Psi_{2}^{-} \frac{1+\Psi_{1}^{-} \Psi_{3}}{1+\Psi_{3}^{2}} \\
\xi_{+}=\xi(1+\delta)\end{array}$ & $\begin{array}{c}G=\psi-\operatorname{atan} \Psi_{3} \\
L=\psi-\operatorname{atan} \Psi_{1}^{+} \\
V=\Psi_{2}^{+} \frac{1+\Psi_{1}^{+} \Psi_{3}}{1+\Psi_{3}^{2}} \\
\xi_{-}=\xi(1-\delta)\end{array}$ \\
\hline Eq. & Eq. (22), (21) & Eq. (22), (23) \\
\hline
\end{tabular}

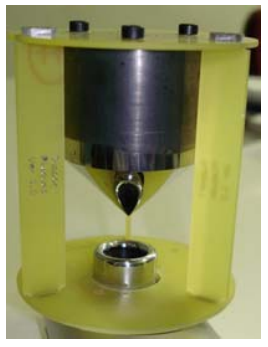

$a$

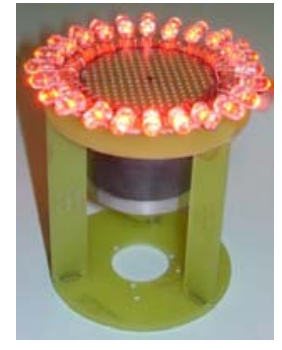

$b$

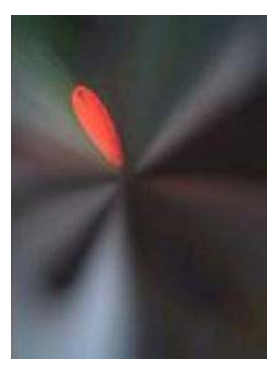

C
Fig. 2. The conic mirror placed above the webcam $(a)$. The circular set of LEDs forming the light source, positioned at the origin $(b)$. The light source used in our experiments as seen through the adopted vision sensor after defocusing $(c)$ : it looks like an ellipse.

$\phi+\psi$ is time independent. On the other hand, with the pure rotation $\mu, \gamma$ and $\psi$ are observable. Therefore, from the values of $\mu$ and $D$ it is possible to determine $\rho$ and from $\psi$ and the sum $\phi+\psi$ it is possible to determine $\phi$. This is exactly what we obtained in our experiment, where the estimation was performed by implementing an Extended Kalman Filter. In particular, a single filter was implemented to estimate the state $[\mu, \gamma, \phi, \rho, \psi]^{T}$. Better results can be achieved by implementing one filter for every robot motion, accordingly to the equations corresponding to every decomposition. In our experiment, the robot performs a long shift at the beginning starting very far from the source of light and ending very close. Then it starts to perform a pure rotation. In figure 3 there is a jump in the estimated values of the three parameters $\phi, \rho$ and $\psi$. This jump occurs exactly when the robot starts to rotate. Observe that during all the experiment, the estimated value of $\phi+\psi$ is almost constant. Indeed, according to our theory, this quantity can be estimated starting from the beginning, i.e. by moving the robot far from the feature and along a straight trajectory. 

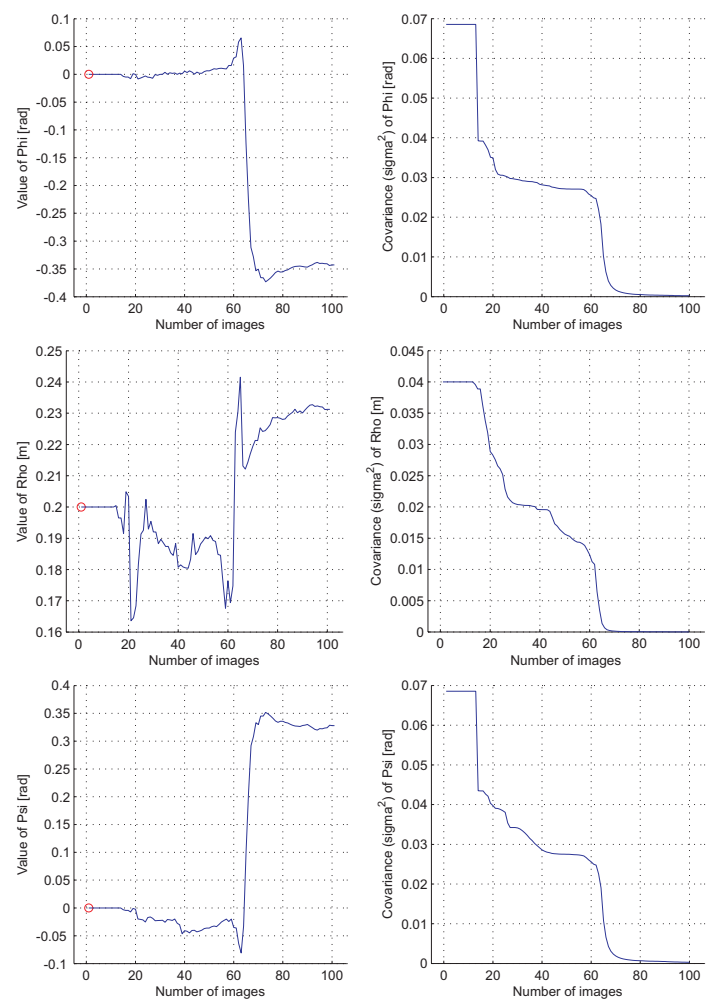

Fig. 3. The estimated $\phi, \rho$ and $\psi$ during the robot motion consisting of a pure shift far from the feature and a pure rotation close to the feature. The adopted units are radians and meters.

\section{CONCLUSIONS}

In this paper a new approach based on the theory of distributions has applied to face a calibration problem in the framework of mobile robotics. The method is very general and could be applied to solve every estimation problem. It exploits all the system Lie symmetries through the Frobenius theorem which is at the heart of the distribution theory [7]. Regarding the considered calibration problem this method allows us to analytically detect the combinations of the calibration parameters which are observable for a given robot trajectory.

We are making more complete experiments by including the odometry calibration as discussed in section $\mathrm{V}$ and by also comparing the performance achievable by implementing a single filter and one filter for every system decomposition. Preliminary results show significant improvement in the second case as expected since all the estimated quantities are observable and the estimation process is consistent.

\section{REFERENCES}

[1] Bicchi A., Pratichizzo D., Marigo A. and Balestrino A., On the Observability of Mobile Vehicles Localization, IEEE Mediterranean Conference on Control and Systems, 1998

[2] Bonnifait P. and Garcia G., Design and Experimental Validation of an Odometric and Goniometric Localization System for Outdoor Robot Vehicles, IEEE Transaction On Robotics and Automation, Vol 14, No 4, August 1998

[3] Borenstein J., Feng L., "Measurement and correction of systematic odometry errors in mobile robots," IEEE Transactions on Robotics and Automation, vol. 12, pp. 869-880, 1996.
[4] Caltabiano D., Muscato G. and Russo F., "Localization and Self Calibration of a Robot for Volcano Exploration" International Conference on Robotics and Automation, pp. 586-591, New Orleans, USA, 2004.

[5] E.M. Foxlin, Generalized Architecture for Simultaneous Localization, Auto-Calibration, and Map building, International Conference on Intelligent Robots and Syrtems, EPFL, Switzerland, October 2002.

[6] Hermann R. and Krener A.J., 1977, Nonlinear Controllability and Observability, IEEE Transaction On Automatic Control, AC-22(5): 728-740

[7] Isidori A., Nonlinear Control Systems, 3rd ed., Springer Verlag, 1995.

[8] Larsen, T.D., Hansen K.L., Andersen N.A. and Ole Ravn, Design of Kalman filters for mobile robots; evaluation of the kinematic and odometric approach, Proceedings of the 1999 IEEE International conference on Control Applications, volume: 2 , 22-27 Aug. 1999, Pages:1021-1026.

[9] A. Martinelli, N. Tomatis, A. Tapus and R. Siegwart, Simultaneous Localization and Odometry Calibration for Mobile Robot, International Conference on Intelligent Robots and Systems, Las Vegas, Nevada, October 2003.

[10] A. Martinelli and R. Siegwart, Observability Analysis for Mobile Robot Localization, International Conference on Inteligent Robot and Systems, Edmonton, Canada, August 2005.

[11] A. Martinelli, D. Scaramuzza and R. Siegwart, Automatic SelfCalibration of a Vision System during Robot Motion, International Conference on Robotics and Automation, Orlando, Florida, April 2006

[12] A. Martinelli, J. Weingarten and R. Siegwart, Theoretical Results on On-line Sensor Self-Calibration, International Conference on Inteligent Robot and Systems, Benjing, China, October 2007.

[13] G.P. Huang, A.I. Mourikis, and S.I. Roumeliotis, "Analysis and improvement of the consistency of extended Kalman filter based Slam", In Proceedings of the 2008 IEEE International Conference on Robotics and Automation (ICRA), pages 473479, May 2008.

[14] Roy N., and Thrun S., Online Self-calibration for Mobile Robots, proceedings of the 1999 IEEE International Conference on Robotics and Automation, 19 May 1999 Detroit, Michigan, pp. 2292-2297.

[15] Scaramuzza D., Martinelli A. and Siegwart R., "Precise Bearing Angle Measurement Based on Omnidirectional Conic Sensor and Defocusing", Proceedings of European Conference on Mobile Robots, ECM R05, Ancona, Italy, September 2005.

[16] H.J. von der Hardt, R. Husson, D. Wolf, An Automatic Calibration Method for a Multisensor System: Application to a Mobile Robot Localization System, Interbational Conference on Robotics and Automation, Leuven, Belgium, May 1998.

\section{APPENDIX}

We apply the rank criterion to prove that the state $[D, \theta, \zeta]$ is observable when satisfying the equations in (14). The Lie derivatives are computed along the vector field $f \equiv\left[\cos \theta,-\frac{\sin \theta}{D}, 0\right]^{T}$ and the observation function is $y=-\theta-\zeta$ which is also the zero order Lie derivative $\left(y=L^{0} y\right)$. The gradient of this function is: $d L^{0} y=[0,-1,-1]^{T}$. Hence, the first order Lie derivative is $L_{f}^{1}=$ $d L^{0} y^{T} f=\frac{\sin \theta}{D}$ whose gradient is: $d L^{1} y=\left[-\frac{\sin \theta}{D^{2}}, \frac{\cos \theta}{D}, 0\right]^{T}$. The second order Lie derivative is $L_{f}^{2}=d L^{1} y^{T} f=-\frac{\sin 2 \theta}{D^{2}}$ whose gradient is: $d L^{2} y=\left[\frac{2 \sin 2 \theta}{D^{3}},-\frac{2 \cos 2 \theta}{D^{2}}, 0\right]^{T}$. By computing the following determinant:

$$
\left|\begin{array}{l}
d L^{0} y \\
d L^{1} y \\
d L^{2} y
\end{array}\right|=\frac{2}{D^{4}} \sin \theta
$$

we know that the previous gradients are independent in all the configuration space where $\theta \neq k \pi, k=\ldots,-2,-1,0,1,2, \ldots$. This proves the local weak observability of $[D, \theta, \zeta]$. 